CentroSur

Social Science Journal

\section{Implementación de un módulo de práctica de filtros digitales FIR para la Carrera Teleinformática de la Universidad de Guayaquil: caso práctico}

Case study: Implementation of a practice module of FIR digital filters for the Teleinformatics Career at the University of Guayaquil
Centro Sur.

Social Science Journa

Julio - Diciembre Vol 4 No 2 http://centrosureditorial.com/ind ex.php/revista elSSN: 2600-5743

revistacentrosur@gmail.com Recepción: 5 noviembre 2019 Aprobación 9 febrero 2020 Pag 59 - 73

Atribución/ReconocimientoNoComercial-Compartirlgual 4.0 Licencia Pública Internacional CC BY-NC-SA 4.0 https://creativecommons.org/lice nses/by-nc-sa/4.0/legalcode.es

\author{
Neiser Ortiz Mosquera ${ }^{1}$ \\ Gregorio Rodríguez Suárez² \\ Ximena Trujillo Borja ${ }^{3}$
}

\section{Resumen}

El presente trabajo de investigación tiene como objetivo de implementar un Módulo de Práctica de Filtros Digitales FIR para la Carrera Teleinformática de la Universidad de Guayaquil. Se utilizó el método bibliográfico para determinar que el método matemático de ventana es el más adecuado para el diseño de los filtros digitales FIR porque permitió usar hardware programable para implementar los diferentes tipos de filtros digitales, para la implementación del módulo se usó el método experimental y por último, se utilizó el método indagación para determinar la usabilidad del módulo a través de encuesta que se aplicó a los 13 estudiantes de octavo semestre que estaban registrado en la materia de
Simulación de Sistema de la Carrera Ingeniería de Teleinformática de la Universidad de Guayaquil en el período académico Ciclo 2019 - 2020 CII. Se realizó una comparación de la respuesta de salida de cada filtro implementado experimentalmente del

\footnotetext{
${ }^{1}$ Magister en Gerencia de Redes y Telecomunicaciones, Docente titular. Universidad de Guayaquil. Guayaquil Ecuador. ORCID https://orcid.org/0000-0002-1051-6102. Email.neiser.ortizm@ug.edu.ec ID Scopus 57196439723 2 Ingeniero Teleinformática, Tesista, Universidad de Guayaquil. Guayaquil - Ecuador ORCID. https://orcid.org/0000-0002-2236-4820 EMAIL. gregorio.rodriguezs@ug.edu.ec. Google Académico.

https://scholar.google.es/citations?hl=es\&user=vY 5 YTQAAAAJ

3 Magister en Gerencia de Redes y Telecomunicaciones, Docente titular, Universidad de Guayaquil. Guayaquil Ecuador ID ORCID https://orcid.org/0000-0003-20935906. Email . ximena.trujillob@ug.edu.ec. ID Scopus 57196440324
} 
módulo con la respuesta de salida simulada observándose un mínimo error entre ellos y que la respuesta de salida experimental tiene una mayor ganancia, también se monitoreo el tiempo de ejecución de la práctica obteniéndose como promedio total 84,75 minutos de la implementación y por último se obtuvo que más del $90 \%$ de los encuestados indicó que el módulo era un sistema útil, intuitivo, amigable, eficiente y fiable. Se concluye que la implementación del Módulo tiene una respuesta excelente de salida experimental, optimiza tiempo de práctica y que tuvo una buena aceptación de los estudiantes.

Palabras clave: Filtro digitales, módulo, práctica, FIR

\section{Abstract}

The present work of investigation has like objective to implement a Module of Practice of FIR Digital Filters for the Teleinformatics Career of the University of Guayaquil. The bibliographic method was used to determine that the window mathematical method is the most suitable for the design of the FIR digital filters because it allowed the use of programmable hardware to implement the different types of digital filters. For the implementation of the module, the experimental method was used and finally the research method was used to determine the usability of the module through a survey that was applied to the 13 students of the eighth semester that were registered in the subject of System Simulation of the Teleinformatics Engineering Career of the University of Guayaquil in the academic period Cycle 2019 - 2020 CII. A comparison was made between the output response of each filter implemented experimentally in the module and the simulated output response, with a minimum error between them. The experimental output response had a higher gain, and the practice execution time was also monitored, obtaining a total average of 84.75 minutes of implementation. It is concluded that the implementation of the Module has an excellent response of experimental output, optimizes time of practice and that it had a good acceptance of the students.

Key words: Digital Filter, module, practice, FIR

\section{Introducción}

El estudiante desarrolla el proceso de análisis y compresión con la actividad experimental. Las herramientas o equipos adecuados en la actividad experimental los prepara en el ámbito profesional aumentado confianza, desarrolla habilidades, y 
destrezas que permitirán aplicarla en el mundo laboral. Los estudiantes que realizan actividades experimentales académicas dentro de la carrera profesional tendrán la capacidad adecuada para ser competitivos en su carrera profesional en el ámbito laboral. (López \& Tamayo, 2012)

El trabajo de (Urrea, Niño, García, \& Alvarado, 2013) indica también que tener laboratorios completos, equipados y estructurado correctamente permiten ser un mecanismo de enseñanza-aprendizaje. Los trabajos de investigación de (ALVARADO, ANTUNEZ, PIRELA, \& PRIETO, 2011) y (FLORES, ÁVILA, \& OLVERA, 2017) indican la importancia de las prácticas que realizan los estudiantes en el laboratorio permiten al estudiante implementar prácticas para comprobar, analizar y comparar los resultados experimentales con los resultados teóricos permitiendo así consolidar el proceso de aprendizaje. Es importante que en el laboratorio el estudiante pueda usar software o hardware (Ruiz Silva, 2016) que emule el comportamiento de sistemas reales permitiendo así un acercamiento real de la dinámica de las variables cuyos valores se pueden medir y controlar.

La carrera de Teleinformática de la Universidad de Guayaquil tiene la materia de Simulación de Sistemas que es una materia netamente práctico del área de las Telecomunicaciones dentro de su malla curricular. El laboratorio donde se da dicha materia no tiene los recursos necesarios para realizar las debidas prácticas que necesitan los estudiantes que dificulta así el proceso de aprendizaje. Por las dificultades mencionadas anteriormente se decidió abrir un proyecto de Fondos Concursables de Investigación (FCl) en la Universidad de Guayaquil en el cual fue aprobado en Sesión del Honorable Consejo Universitario de la Universidad de Guayaquil mediante Resolución RCU-SO-04-121-04-2018. (Universidad de Guayaquil, 2018)

Los trabajos (Alvarado Nieves, 2019), (Ramírez Macías, 2019), (Pillasagua Oviedo, 2019) y (Pacheco Santana, 2019) demuestran que con la implementación de módulos electrónicos para la realización de prácticas experimentales se obtiene resultados confiables de sistemas de modulaciones y demodulaciones de señales analógicas y digitales y también en la optimización de tiempo y recurso. Los trabajos anteriormente mencionados cumplen el objetivo general del proyecto $\mathrm{FCl}$ que es de desarrollar un espacio de aprendizaje práctico del área de telecomunicaciones, utilizando nuevas herramientas de bajo costo que permitan la mejora del conocimiento de los estudiantes tanto para su desempeño académico como para investigación.

El presente trabajo tiene como objetivo general de implementar un Módulo de Práctica de Filtros Digitales para la Carrera Teleinformática dentro de los laboratorios de Networking/Telecomunicaciones. La implementación del módulo permitirá que todos los elementos que se necesitan para realizar una práctica de 
Filtros Digitales estarán disponible en un circuito impreso para que los estudiantes tengan más tiempo para el análisis de los resultados experimentales.

El trabajo de investigación (Álvarez, Lindig, \& Martínez, 2008) indica que para la implementación de los filtros digitales solo se realiza con señales discretas debido que son algoritmos matemáticos. También (Emilia, 2009) indica que los filtros digitales están diseñados con algoritmos matemáticos de alta complejidad permitiendo la implementación de estos filtros tanto en hardware como software. El trabajo (Delgado Vallejo, 2017) demuestra que los filtros digitales se pueden implementar en tecnología programables como los microcontroladores debido que estos filtros tienen la predisposición de funcionar en hardware como software.

Dentro de los diferentes tipos de filtros digitales el más estable con fase lineal y fácil de diseñar son los filtros digitales de Respuesta finita al impulso (FIR). EI FIR tiene la ventaja de que más números de coeficientes se halle mejor será la resolución de salida de la señal pero se ocasiona un uso mayor de memoria de los sistemas programables. (Cordero, 2006)

\section{Materiales y métodos}

Para el presente proyecto se utilizó el método bibliográfico y experimental. El método bibliográfico permitió conocer que el algoritmo matemático más adecuado era método de ventana (Álvarez, Lindig, \& Martínez, 2008) y que el hardware un microcontrolador PIC (Delgado Vallejo, 2017). Antes de realizar la implementación del módulo del filtro digital FIR el método experimental (Pantoja Rodríguez \& Mateo Washbrum, 2019) (Mena Saucedo \& González Contreras, 2018) permitió conocer que el software más idóneo para la simulación del diseño del filtro FIR era el Proteus (Electronic, 2020) y también ayudo en la selección adecuada de los elementos electrónicos.

Para la realización del módulo electrónico del Filtro FIR en este trabajo de investigación, se desarrolló las siguientes actividades como la realizacón del algoritmo usando el método de ventana, programación en lenguaje $C$ del microcontrolador PIC, simulación en software Proteus del circuito electrónico y por último la creación en Ares (herramienta de Proteus) de la placa de circuito impreso. También se utilizó el método indagación (Massa, De Giusti, \& Pesado, 2012) para determinar la usabilidad del módulo electrónico del Filtro FIR implementado con el objetivo de recabar información relativa a la interacción de los usuarios con el sistema y la percepción subjetiva de estos sobre la experiencia de manejo. La encuesta fue el instrumento de aplicación del método de indagación que se aplicó a los 13 estudiantes de octavo semestre que estaban registrado en la materia de Simulación de Sistema de la Carrera Ingeniería de Teleinformática de la Universidad 
de Guayaquil en el período académico Ciclo 2019 - 2020 Cll en el laborarorio de Networking/Telecomunicaciones.

Antes de realizar la implementación del módulo electrónico del filtro digital FIR, primero se debe explicar el modelo matemático que se usó ya que un filtro digital es un algoritmo que permite o rechaza un rango de frecuencias de una señal de entrada. Con la ayuda del método de la transformada de Fourier se diseñó los diferentes tipos de filtros digital FIR. En la tabla 1 y 2 se observa los diferentes tipos de fórmulas para el cálculo de los coeficientes de los filtros digitales y Ventanas. (Jarrín Ochoa, 2016)

Tabla 1. Descripción matemática de los coeficientes $h(n)$ de los diferentes tipos de filtros FIR

\begin{tabular}{l|l|c|l|l} 
Muestras & \multicolumn{4}{l}{ Coeficiente $\mathbf{h}(\mathbf{n})$ de un filtro digital FIR } \\
\hline $\begin{array}{l}\mathrm{M} \\
-\frac{\mathrm{M}}{2}<\mathrm{n}<\end{array}$ & $\begin{array}{l}\text { Pasa } \\
\text { Bajos }\end{array}$ & Pasa Altos & Pasa Banda & Rechaza Banda \\
\hline $\mathrm{n}=0$ & $\frac{w_{c}}{\pi}$ & $1-\frac{w_{c}}{\pi}$ & $\frac{w_{c 2}-w_{c 1}}{\pi}$ & $1-\frac{w_{c 2}-w_{c 1}}{\pi}$ \\
\hline $\mathrm{n} \neq 0$ & $\frac{\sin \left(w_{c} n\right)}{\pi \mathrm{n}}$ & $\frac{-\sin \left(w_{c} n\right)}{\pi \mathrm{n}}$ & $\frac{\sin \left(w_{c 2} n\right)-\sin \left(w_{c 1} n\right)}{\pi \mathrm{n}}$ & $\frac{\sin \left(w_{c 1} n\right)-\sin \left(w_{c 2} n\right)}{\pi \mathrm{n}}$
\end{tabular}

En la tabla 1 se observa el modelo matemático para el cálculo de los coeficientes $\mathrm{h}(\mathrm{n})$ para cada uno de los filtros digitales que se diseñó. Donde $\mathrm{n}$ representa los términos o intervalos del ancho de banda de la señal y $\mathrm{M}$ representa el orden de los sistemas de filtros FIR.

En la ecuación 1 se observa la fórmula de la frecuencia de corte $\left(w_{c}\right)$ que cuya unidad es el radian. $W_{c}$ es un término de relevancia para el cálculo de la frecuencia de corte $\left(f_{c}\right)$ de cada uno de los filtros digital implementado. $W_{c}$ también está relacionado con la frecuencia de muestreo $\left(f_{\mathrm{s}}\right)$, ya que la señal analógica que se desea filtrar se tiene que digitalizar aplicando el teorema de Nyquist. Las frecuencias de $f_{c} y f_{s}$ tienen de unidad el Hertzios.

$$
w_{c}=2 * \pi * \frac{f_{c}}{f_{s}} \quad \text { Ecuación } 1
$$


Tabla 2. Descripción matemática de los coeficientes $w(n)$ de los diferentes tipos de ventanas

Coeficiente w(n) de una Ventana fija

\begin{tabular}{l|l|l|l|l}
\hline $\begin{array}{l}\text { Muestr } \\
\text { as }\end{array}$ & Rectangular & Hamminig & Hanning & Blackman \\
\hline $\begin{array}{l}0 \leq \mathrm{n} \leq \\
\mathrm{M}\end{array}$ & 1 & $\frac{1-\cos \left(\frac{2 \pi n}{M}\right)}{2}$ & $\frac{27-23 \cos \left(\frac{2 \pi n}{M}\right)}{50}$ & $\frac{21-25 \cos \left(\frac{2 \pi n}{M}\right)+4 \cos \left(\frac{4 \pi n}{M}\right)}{50}$ \\
\end{tabular}

En la tabla 2 se observa el modelo matemático para el cálculo de los coeficientes de las ventanas $w(n)$. Las ventanas trabajan juntas con las funciones de transferencia $h(n)$ como indica la ecuación 2, el objetivo de este método de ventanas es suavizar y mejorar la respuesta en frecuencia de los filtros FIR. En la ecuación 2 se observa la fórmula General de la función de transferencia de salida $H(n)$ de un filtro digital FIR.

\section{Filtro multi banda}

$$
\mathrm{H}(\mathrm{n})=\mathrm{h}(\mathrm{n}) \cdot \mathrm{w}(\mathrm{n}) \quad \text { Ecuación } 2
$$

También se implementó el filtro multi banda ya que el modelo matemático escogido permite implementar filtros de más alta complejidad dentro del módulo electrónico. Este filtro permite el paso a frecuencias múltiples en un solo diseño de filtro, cabe recalcar que el resultado de la última frecuencia calculada debe ser igual a $\pi$ este diseño de filtro son recomendables para sistemas que requieren hacer ecualizaciones sobre la señal de entrada. La función de transferencia para el cálculo de los coeficientes para este filtro se observa en la tabla 3.

Tabla 3. Descripción matemática de los coeficientes $h(n)$ del filtroFIR multi banda

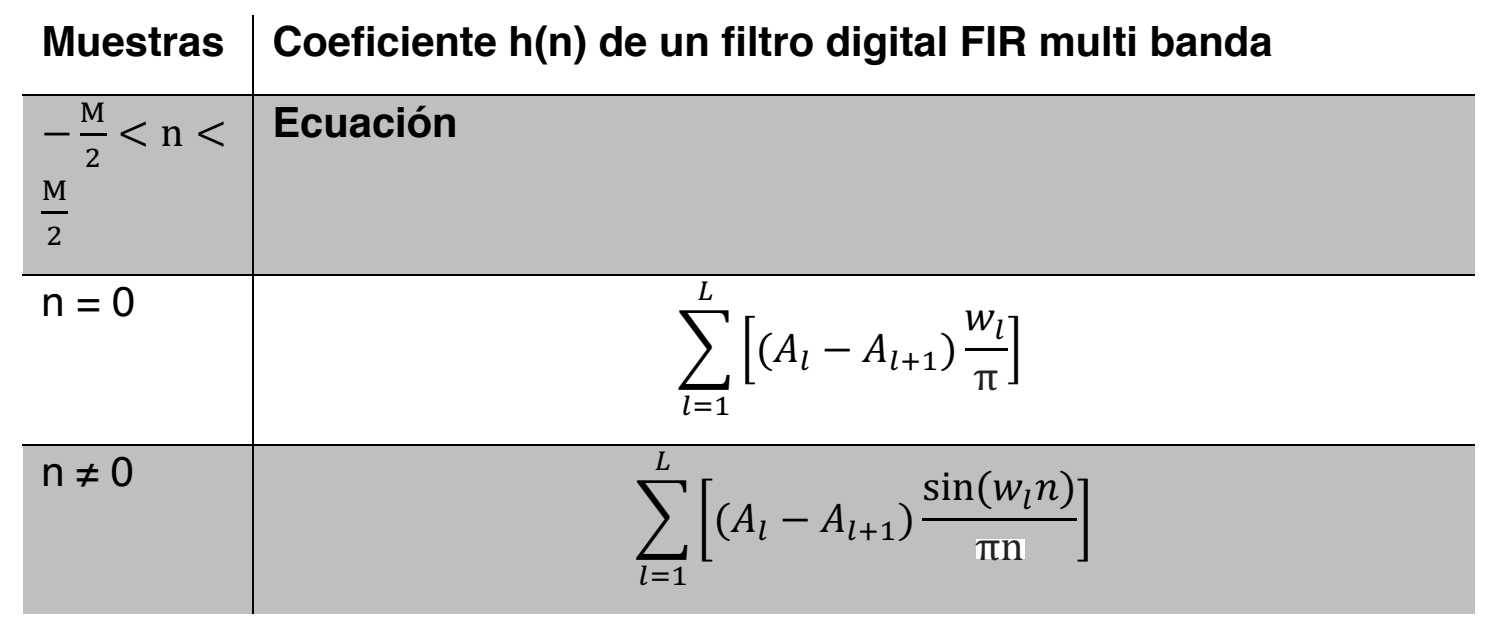




\section{Diseño de circuito electrónico del módulo del filtro digital FIR}

Se realizó la simulación del diseño electrónico del módulo del Filtro FIR con el software Proteus y se usó como elemento principal del diseño un microcontrolador PIC 18F452 (Microchip, 2019) con una frecuencia de reloj de $10 \mathrm{MHz}$ activando desde Mikroc Pro Pic (Mikroe, 2019) la fuente de reloj de hs pll donde internamente el PIC multiplica por un factor de cuatro la frecuencia externa teniendo al final un total de $40 \mathrm{MHz}$. Esto es necesario para tratamiento digital de la señal analógica que va a ingresar al PIC dando como resultado 10 Millones de instrucciones por segundo por lo que cada cuatro ciclos de reloj cumplen con una instrucción. Como elementos complementarios al diseño electrónico se usó un teclado matricial 4x4 para ingresar los coeficientes de la función de transferencia de salida $\mathrm{H}(\mathrm{n})$ de los filtros digitales. También se usó pantalla LCD para visualizar el encendido, la solicitud del ingreso de los coeficientes $\mathrm{H}(\mathrm{n})$ y finalización del mismo. Para el funcionamiento correcto de la pantalla LCD se incorporó el circuito integrado PCF8574 (Texas Instruments, 2019), que es un expansor de entradas/salidas compatible con el microcontrolador PIC 18F452, que permite convertir una comunicación paralela a una comunicación I2C. Y por último se utiliza un convertido Digital a Analógico R2R en la salida del diseño electrónico del filtro cuyos componentes principales son resistencias de $10 \mathrm{~K} \Omega$ y $20 \mathrm{~K} \Omega$, un capacitor $100 \mathrm{nF}$ y un circuito integrado OPAM LM741. En la figura 1 se observa el esquema general del circuito electrónico diseñado.

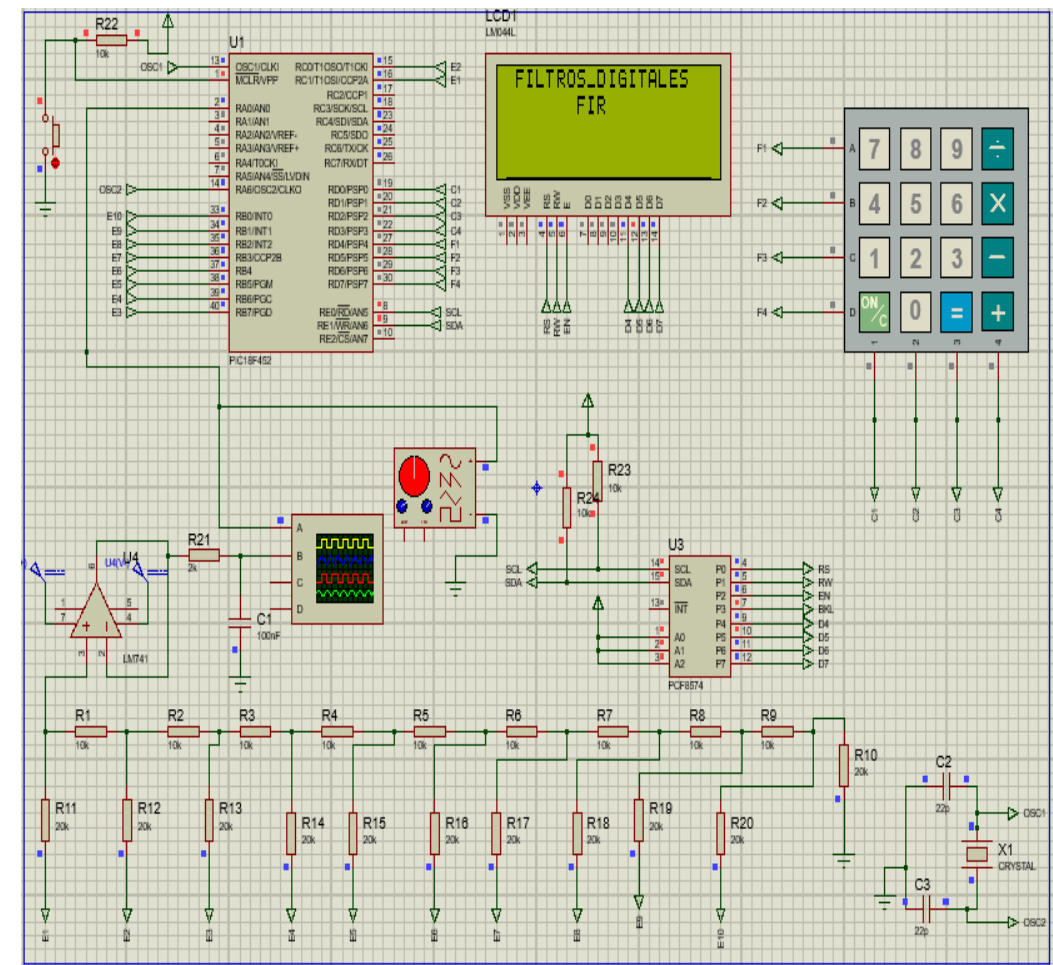

Figura 1. Esquema general del circuito electrónico de filtros digitales FIR. 


\section{Programación del microcontrolador PIC 18F452}

A continuación se detalla la programación del microcontrolador PIC 18F452 en el diagrama de flujo que se detalla en la figura 2 .

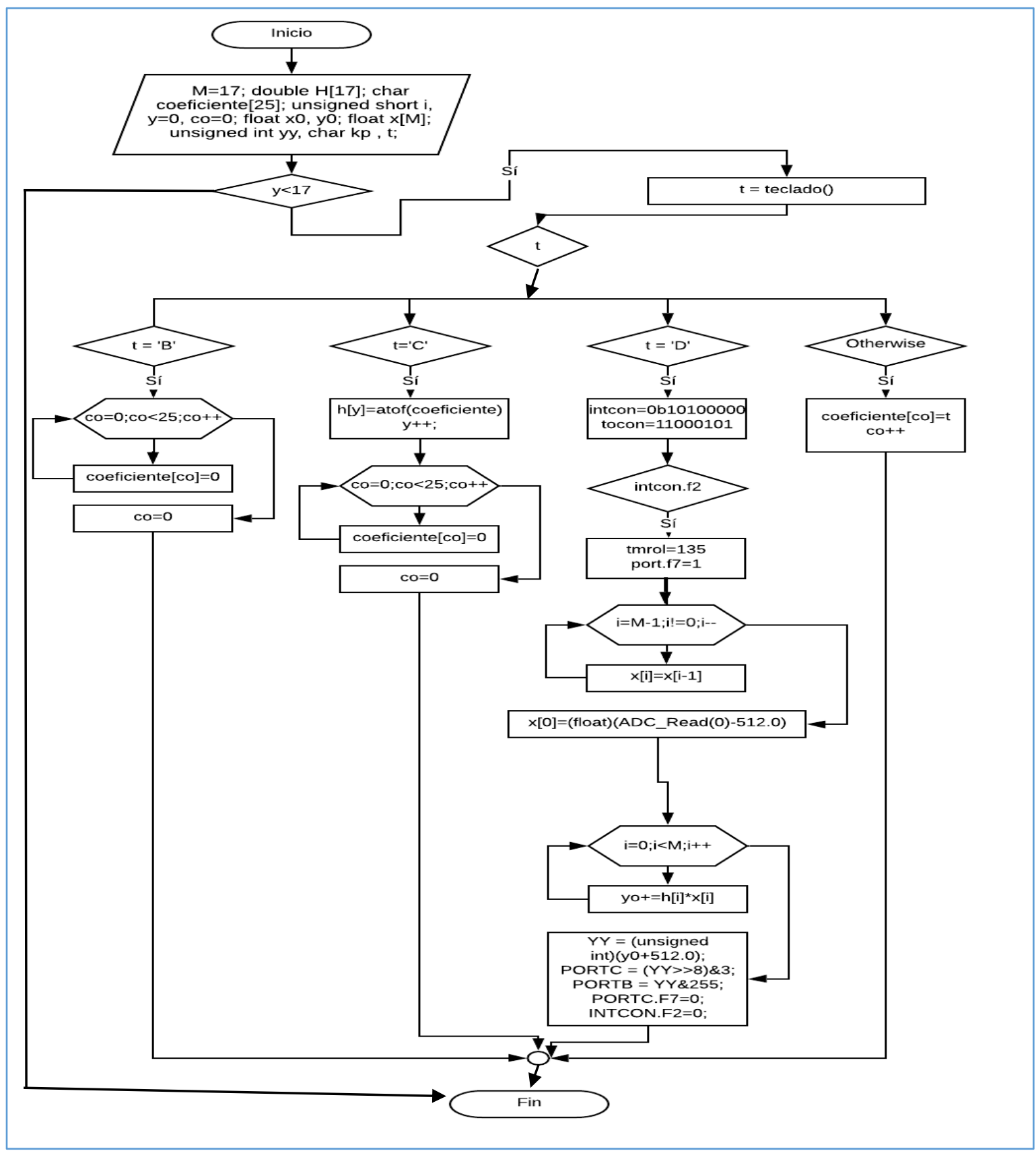

Figura 2. Diagrama de flujo de la programación del microcontrolador PIC 18F452.

El diagrama de flujo indica que los coeficientes de la función de transferencia de salida $H(n)$ se ingresa a través del teclado mostrando la versatilidad que tiene el sistema ya que no necesita estar reprogramando el PIC cada vez que se desee implementar un nuevo tipo de Filtro digital FIR. Los valores de los coeficientes 
dependen del filtro y la ventana que se desea implementar en el módulo electrónico. El número de muestras máximo que soporta el microcontrolador es $\mathrm{M}=17$ debido al límite de la memoria que posee.

Creación de la placa del circuito impreso del módulo electrónico del Filtro Digital FIR

Como el diseño electrónico se hizo en Proteus los componentes del módulo del Filtro Digital FIR ya están listo para la creación de la placa del circuito impreso en el software Ares. Se procedió a realizar el auto ruteo dentro del Software Ares, para que las pistas del circuito impreso vaya de acuerdo al diseño como se trabajó en Proteus. Por último para imprimir la placa del circuito impreso se guardó en formato Gerber. En la figura 3 se observa dicho diseño.

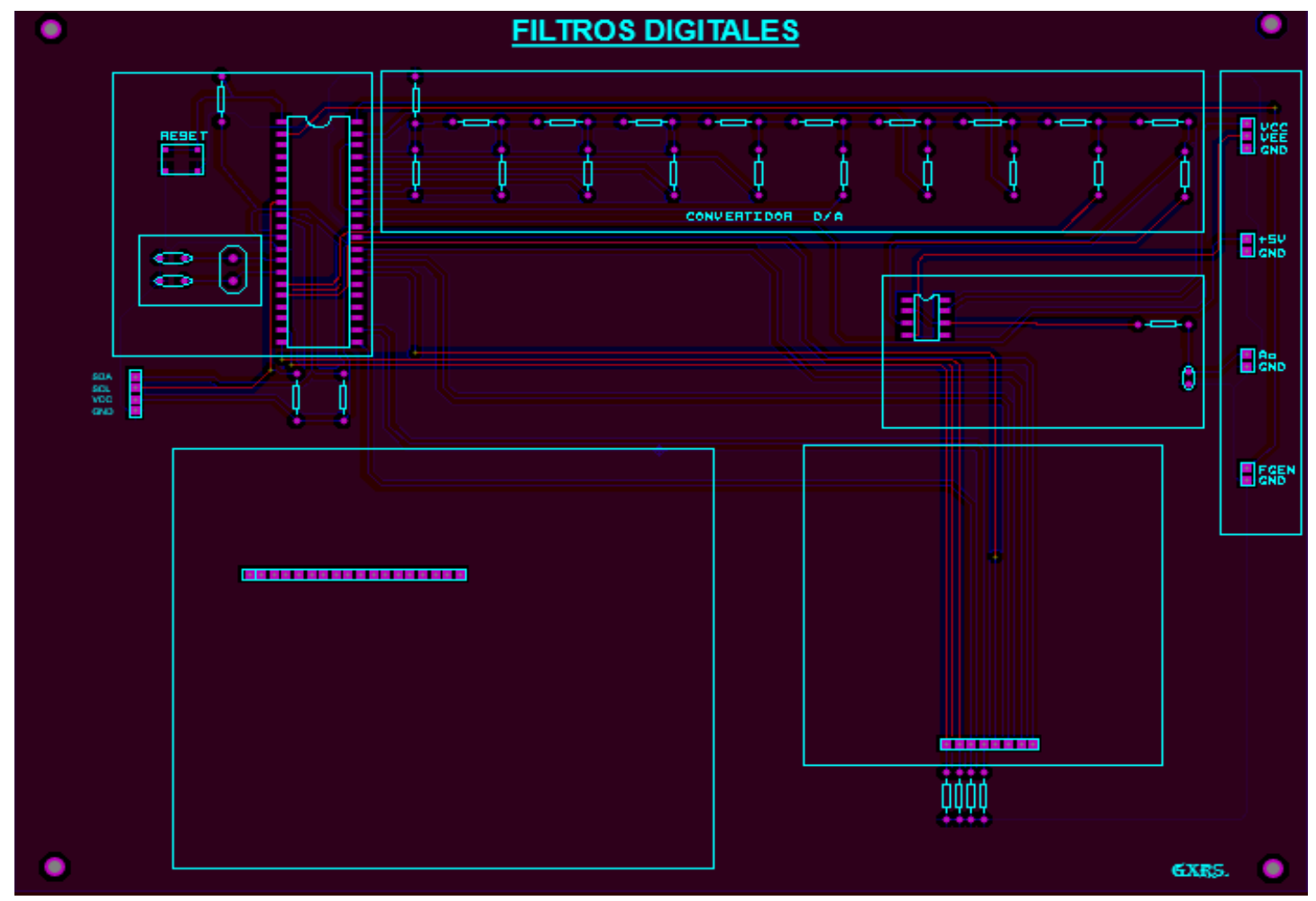

Figura 3. Diseño de la placa del circuito impreso del módulo electrónico del filtro digital FIR

\section{Resultados}

Después de hacer el Módulo de Práctica de Filtros Digitales se realizó un experimento práctico con los estudiantes registrados en la materia de Simulación de Sistemas para verificar el funcionamiento del mismo. Los resultados obtenidos de cada filtro digital implementado en el módulo se observa en la tabla 4. 
Tabla 4. Diagrama de Bode de los diferentes tipos de Filtros Digitales FIR del módulo

Diagrama de Bode de los Filtros Digitales FIR

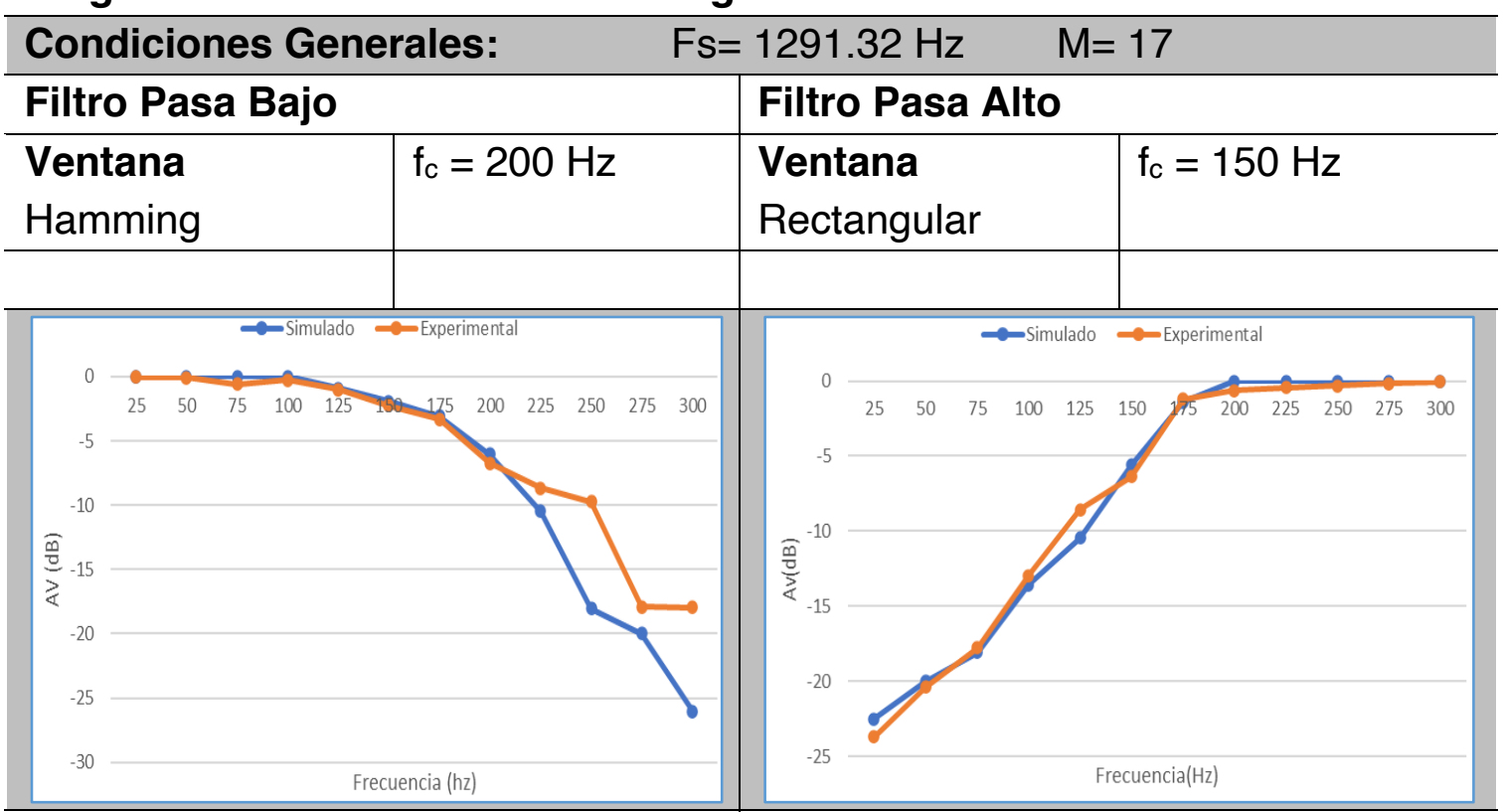

\section{Filtro Pasa Banda}

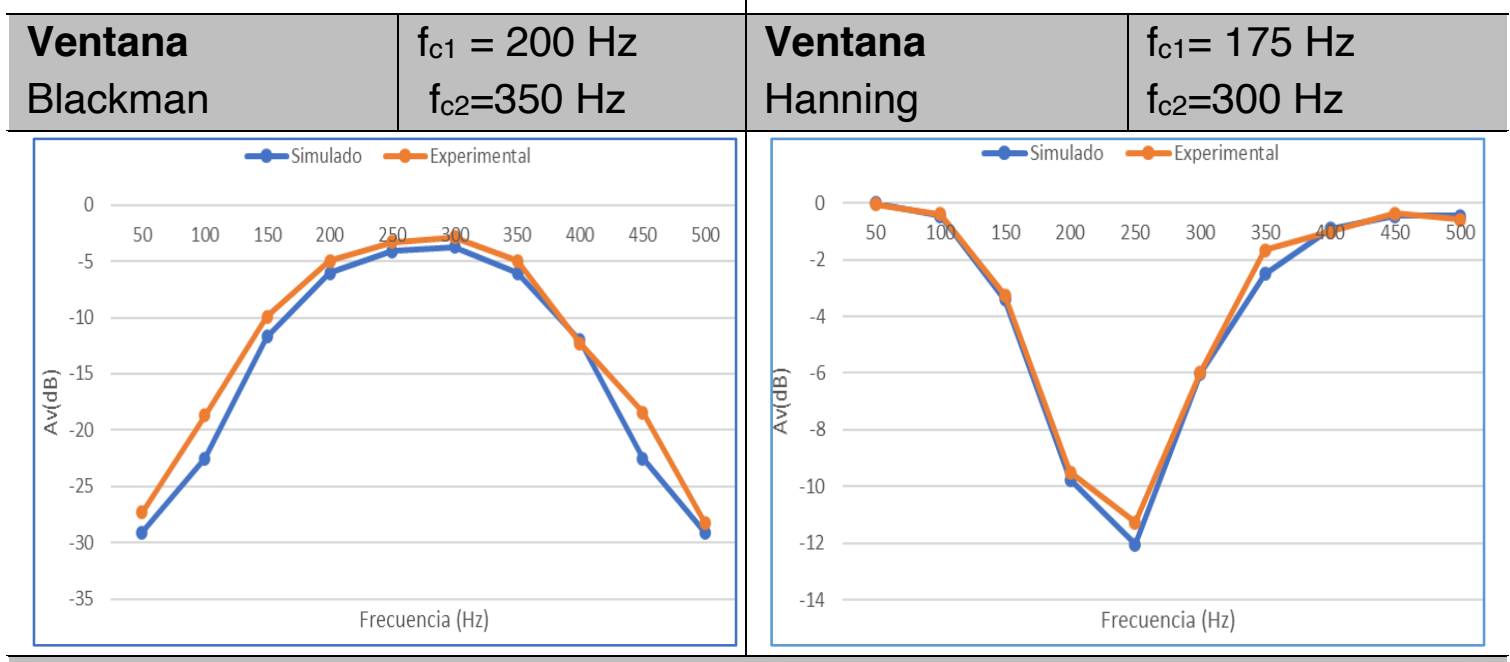

Filtro Multi Banda 


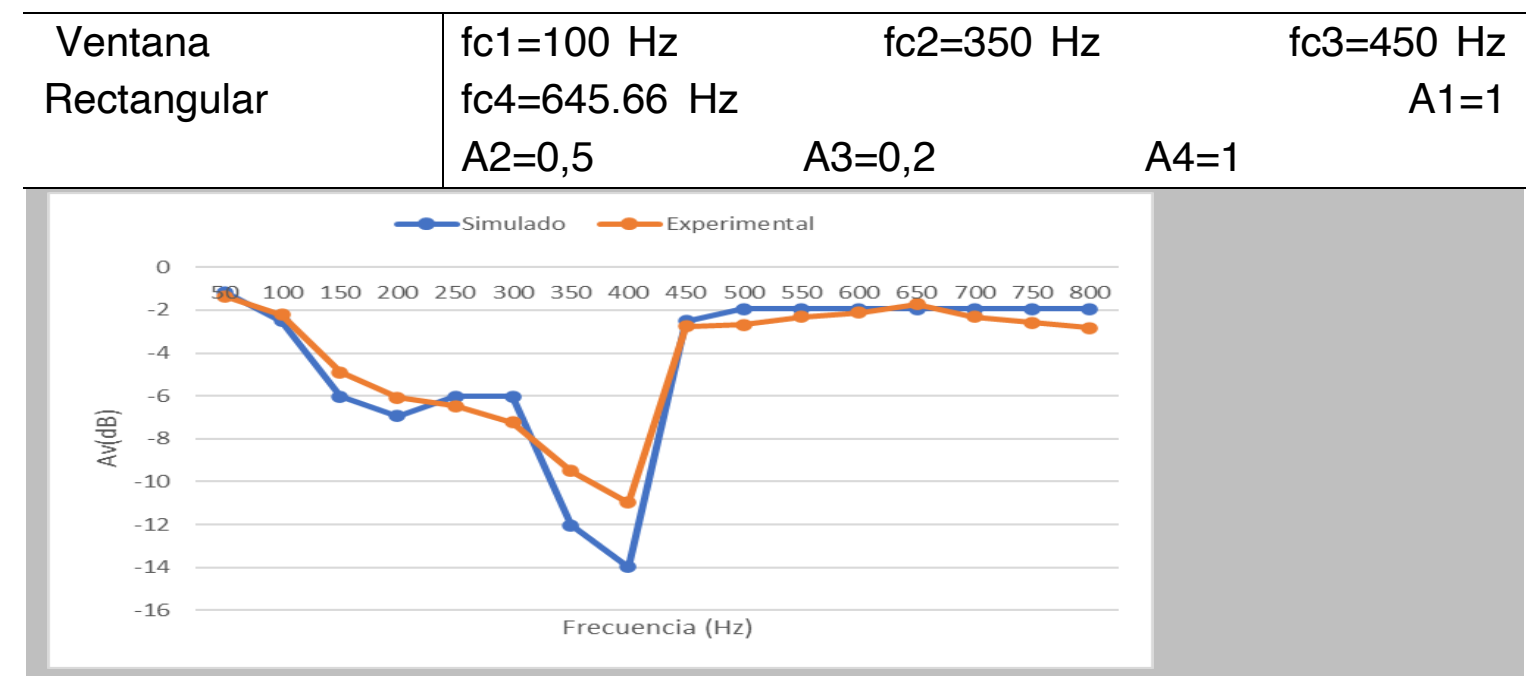

Como se observa en la tabla 4, se realizó una comparación de la respuesta de salida de cada filtro implementado experimentalmente del modulo de Práctica de Filtros Digitales con la respuesta de salida simulada observándose un mínimo error entre ellos y también se observa que la respuesta de salida experimental tiene una mayor respuesta de salida con respecto con lo simulado.

También se monitoreo el tiempo de ejecución de la práctica experimental que realizaban los estudiantes de cada filtro digital implementado en el Módulo de Práctica de Filtros Digitales, como se muestra en la tabla 5.

Tabla 5. Monitoreo del Tiempo del experimento

\begin{tabular}{l|l|l|l|l|l} 
Grupos & $\begin{array}{l}\text { Filtro Pasa } \\
\text { Bajo }\end{array}$ & $\begin{array}{l}\text { Filtro } \\
\text { Pasa Alto }\end{array}$ & $\begin{array}{l}\text { Filtro } \\
\text { Pasa } \\
\text { Banda }\end{array}$ & $\begin{array}{l}\text { Filtro } \\
\text { Rechaza } \\
\text { Banda }\end{array}$ & $\begin{array}{l}\text { Filtro Multi } \\
\text { Banda }\end{array}$ \\
\hline 1 & $15 \mathrm{~min}$ & $18 \mathrm{~min}$ & $14 \mathrm{~min}$ & $17 \mathrm{~min}$ & $18 \mathrm{~min}$ \\
\hline 2 & $14 \mathrm{~min}$ & $16 \mathrm{~min}$ & $16 \mathrm{~min}$ & $16 \mathrm{~min}$ & $19 \mathrm{~min}$ \\
\hline 3 & $17 \mathrm{~min}$ & $14 \mathrm{~min}$ & $17 \mathrm{~min}$ & $19 \mathrm{~min}$ & $23 \mathrm{~min}$ \\
\hline 4 & $16 \mathrm{~min}$ & $15 \mathrm{~min}$ & $19 \mathrm{~min}$ & $15 \mathrm{~min}$ & $21 \mathrm{~min}$ \\
\hline Promedio & 15,5 & 15,75 & 16,5 & 16,75 & 20,25
\end{tabular}

Como se observa en la tabla 5 el promedio de la implementación experimental de cada filtro está entre 15 a 20 minutos dando como promedio total 84,75 minutos de la implementación de todos filtros digitales en el módulo.

Por último se realizó una encuesta de la usabilidad del módulo a los estudiantes como se observa en la tabla 6. 
Tabla 6. Encuesta de la usabilidad del módulo electrónico del Filtro FIR

\begin{tabular}{l|l|l|l|l|l}
$\begin{array}{l}\text { Pregunta } \\
\text { Escala }\end{array}$ & Útil & Intuitivo & Amigable & Eficiente & Fiable \\
\hline $\begin{array}{l}\text { Totalmente } \\
\text { de acuerdo }\end{array}$ & $32 \%$ & $17 \%$ & $25 \%$ & $29 \%$ & $32 \%$ \\
\hline De acuerdo & $62 \%$ & $65 \%$ & $67 \%$ & $66 \%$ & $64 \%$ \\
\hline $\begin{array}{l}\text { En } \\
\text { desacuerdo }\end{array}$ & $4 \%$ & $17 \%$ & $6 \%$ & $3 \%$ & $3 \%$ \\
\hline $\begin{array}{l}\text { Totalmente } \\
\text { en } \\
\text { desacuerdo }\end{array}$ & $2 \%$ & $1 \%$ & $2 \%$ & $2 \%$ & $1 \%$ \\
\hline
\end{tabular}

Como muestra la tabla 6 los resultados de la usabilidad del módulo son favorables mostrando que el $94 \%$ de los encuestados indica que es útil para realizar las prácticas experimentales de filtros digitales. Con respecto a la interacción de los usuarios que tuvieron con el módulo se determinó que el 92\% de los encuestados piensan que es un sistema amigable y que el $82 \%$ indica que es un sistema intuitivo. También se observa que el $96 \%$ de los encuestados piensan que el módulo es un sistema fiable en la respuesta de salida que genera y además el $95 \%$ indica que es un sistema eficiente en su funcionamiento.

\section{Conclusiones}

Gracias al método de ventana se puede cambiar el tipo de filtro en el modulo de Práctica de Filtros Digitales, con sólo hallar los coeficientes adecuado y correspondiente para cada uno de ellos. Además este módulo tiene como ventaja de usar hardware programable para implementar los diferentes tipos de filtros digitales evitando cambios de componentes o materiales electrónicos.

Después de hacer el Módulo de Práctica de Filtros Digitales se realizó una comparación de la respuesta de salida de cada filtro implementado experimentalmente con la respuesta de salida simulada observándose un mínimo error entre ellos y también se concluye que la respuesta de salida experimental tiene una mayor respuesta de salida con respecto con lo simulado.

También se monitoreo el tiempo de ejecución de la práctica experimental que realizaban los estudiantes de cada filtro digital implementado en el Módulo de 
Práctica de Filtros Digitales, determinando que el promedio de la implementación experimental de cada filtro está entre 15 a 20 minutos aproximadamente dando como promedio total 84,75 minutos de la implementación de todos filtros digitales.

Se determinó a través de la encuesta de la usabilidad del módulo que el $94 \%$ de los encuestados indica que es útil para realizar las prácticas. Con respecto a la interacción de los usuarios que tuvieron con el módulo se determinó que el 92\% de los encuestados piensan que es un sistema amigable y que el $82 \%$ indica que es un sistema intuitivo. También se observa que el $96 \%$ de los encuestados piensan que el módulo es un sistema fiable en la respuesta de salida que genera y además el $95 \%$ indica que es un sistema eficiente en su funcionamiento. Con todo lo anteriormente mencionado el Módulo de Práctica de Filtros Digitales tuvo una buena aceptación de parte de los estudiantes por ser un sistema útil, intuitivo, amigable, eficiente y fiable.

\section{Referencias}

Alvarado Nieves, J. G. (2019). Implementación de un módulo de práctica FM. Universidad de Guayaquil, Guayaquil. Obtenido de http://repositorio.ug.edu.ec/handle/redug/46676

ALVARADO, Y., ANTUNEZ, J., PIRELA , X., \& PRIETO, A. (Enero-Abril de 2011). Metodología para prácticas en laboratorios de diseño mecánico. Actualidades Investigativas en Educación, XI(1), 1-18. Obtenido de http://www.redalyc.org/pdf/447/44718060006.pdf

Álvarez, C. J., Lindig, B. K., \& Martínez, R. G. (2008). Implementación de Filtros Digitales Tipo FIR en FPGA. Polibits, 83-87.

Cordero, J. A. (2006). Implementacio de filtros digitales en controles digitales de señal. cuenca.

Delgado Vallejo, J. E. (2017). Diseño de un algoritmo para implementar filtros digitales en plataformas basadas en microcontroladores PIC. (Tesis Pregrado). Universidad Privada Antenor Orrego, Perú. Obtenido de http://repositorio.upao.edu.pe/handle/upaorep/3609

Electronic, I. (2020). proteo. Obtenido de www.labcenter.com

Emilia, G. G. (2009). Introducción al filtrado digital. researchgate, 1-14.

FLORES, G., ÁVILA, R., \& OLVERA, R. (Enero-Junio de 2017). La importancia de los laboratorios en la formación del ingeniero. Revista Electrónica.

Humanidades, Tecnología y ciencia IPN(16). Obtenido de http://revistaelectronica-ipn.org/Contenido/17/TECNOLOGIA_17_000461.pdf

Jarrín Ochoa, D. A. (2016). DISEÑO E IMPLEMENTACION DE FILTROS DIGITALES FIR E IIR UTILIZANDO EL MICROCONTROLADOR XMEGA DE ATMEL PARA TRATAMINETO DE SEÑAL DE AUDIO. 
http://dspace.ups.edu.ec/handle/123456789/13087, Quito. Obtenido de http://dspace.ups.edu.ec/handle/123456789/13087

JLCPCB. (2020). https://jlcpcb.com/. jialichuang.

López, R. A., \& Tamayo, A. Ó. (2012). LAS PRÁCTICAS DE LABORATORIO EN LA ENSEÑANZA DE LAS CIENCIAS NATURALES. Latinoamericana de Estudios Educativos, 144-166.

Massa, S. M., De Giusti, A. E., \& Pesado, P. M. (2012). Métodos de evaluación de usabilidad: una propuesta de aplicación en Objetos de Aprendizaje. XIV Workshop de Investigadores en Ciencias de la Computación (págs. 922-926). Misiones: Red de Universidades con Carreras en Informática (RedUNCI).

Mena Saucedo, C., \& González Contreras, B. M. (2018). INSTRUMENTACIÓN Y MONITOREO POR RED INALÁMBRICA DE SENSORES MEDIANTE XBEE PARA UN PROCESO DE POLIMERIZACIÓN. Pistas Educativas(128).

Microchip. (2019). alldatasheet.com. Obtenido de https://pdf1.alldatasheet.com/datasheetpdf/view/90239/MICROCHIP/PIC18F452.html

Mikroe. (2019). mikroC PRO for PIC. Obtenido de https://www.mikroe.com/mikroc-pic Pacheco Santana, M. B. (2019). Implementación de un módulo de práctica BPSK/QPSK usando AD633. Universidad de Guayaquil, Guayaquil. Obtenido de http://repositorio.ug.edu.ec/handle/redug/46707

Pantoja Rodríguez, V., \& Mateo Washbrum, I. (ENERO de 2019). Diseño aplicativo android smart house ITSRV, control domótica vía wifi. Espirales revista multidisciplinaria de investigación, 3(24), 32-41. doi:https://doi.org/10.31876/re.v3i24

Pillasagua Oviedo, S. L. (2019). Implementación de un módulo de práctica PSK/QPSK. Universidad de Guayaquil, Guayaquil. Obtenido de http://repositorio.ug.edu.ec/handle/redug/46711

Ramírez Macías, H. M. (2019). Implementación de un módulo de práctica por desplazamiento de amplitud ASK/AM. Universidad de Guayaquil, Guayaquil. Obtenido de http://repositorio.ug.edu.ec/handle/redug/46713

Ricardo, C. M. (2011). Diseño y simulación de sistemas microcontrolados en lenguaje $C$. colombia.

Ruiz Silva, M. (2016). Diseño de un módulo SCADA de enseñanza práctica con comunicación profibus y PLC'S S7-1200 para el Laboratorio de la Escuela de Ingeniería Electrónica de la UNP. Piura: Universidad Nacional de Piura.

Texas Instruments. (2019). Texas Instruments. Obtenido de http://www.ti.com/lit/ds/symlink/pcf8574.pdf 
Universidad de Guayaquil. (2018). Resoluciones. Obtenido de http://www.ug.edu.ec/resoluciones_consejo_universitario/2018//RCU101\%20A\%20LA\%20150_03-07-2018-111234.pdf

Urrea, Q., Niño, N. A., García, S. I., \& Alvarado, J. P. (2013). DEL AULA A LA REALIDAD. LA IMPORTANCIA DE LOS LABORATORIOS EN LA FORMACIÓN DEL INGENIERO. CASO DE ESTUDIO: INGENIERÍA AERONÁUTICA - UNIVERSIDAD PONTIFICIA BOLIVARIANA. WEEF 